Citation: L. Fugalli (2021) Educaregliadolescenti ai social network. Una ricerca esplorativa su un campione di progetti nelle scuole italiane. Tendenze e modelli di analisi. Media Education 12(1): 17-32. doi: 10.36253/me-9799

Received: September, 2020

Accepted: March, 2021

Published: May, 2021

Copyright: (c) 2021 L. Fugalli. This is an open access, peer-reviewed article published by Firenze University Press (http://www.fupress.com/me) and distributed under the terms of the Creative Commons Attribution License, which permits unrestricted use, distribution, and reproduction in any medium, provided the original author and source are credited.

Data Availability Statement: All relevant data are within the paper and its Supporting Information files.

Competing Interests: The Author(s) declare(s) no conflict of interest.

\section{Educare gli adolescenti ai social network. Una ricerca esplorativa su un campione di progetti nelle scuole italiane. Tendenze e modelli di analisi}

\author{
Social Networks \& Adolescents: The Role of Education. An \\ Exploratory Research on a Sample of Italian School Projects. \\ Trends and Models of Analysis
}

\section{Livia FugALLI}

Dipartimento di Discipline Umanistiche, Sociali e delle Imprese Culturali (DUSIC), Università degli Studi di Parma

E-mail: livia.fugalli@unipr.it

\begin{abstract}
The massive use of social networks by adolescents is a challenging phenomenon which created, in response to a range of psychological and social problems, the development of educational interventions aimed at making adolescents more aware, competent, and responsible. The research identifies and analyzes, on the basis of appropriate documentation, 22 educational projects having the aims and scope mentioned above, which took place in the last decade in several middle and high schools in Italy. Through a set of previously established criteria, an evaluation is carried out in order to describe the most widespread methods applied by such educational interventions. Based on the results, a method of analysis is built through critical comparison, aimed at examining the strengths and weaknesses of the examined projects and at guiding teachers, educators and other specialists in evaluating and designing further projects about the relationship between social networks and adolescents, inside and outside schools.
\end{abstract}

Keywords: media education, social network, adolescents, educational projects.

Riassunto. L'uso massiccio dei social network da parte degli adolescenti ha creato, in risposta ad alcuni problemi dal punto di vista psicologico e sociale, l'elaborazione di interventi educativi finalizzati a rendere gli adolescenti più consapevoli, competenti e responsabili. La ricerca individua e analizza, attraverso la relativa documentazione, 22 progetti educativi riguardanti l'educazione ai social network destinati agli adolescenti, realizzati nell'ultimo decennio in scuole secondarie italiane di primo e secondo grado. L'analisi, attraverso criteri di valutazione stabiliti a priori, vuole far emergere le modalità con le quali sono stati realizzati interventi educativi di tale natura. Sulla base dei risultati, un confronto critico conclusivo intende offrire un modello di analisi che evidenzi punti di forza e di debolezza dei progetti esaminati e che possa orientare insegnanti, educatori e altri specialisti del settore a una futura valutazione e progettazione di ulteriori percorsi inerenti il rapporto tra social network e adolescenti, in contesto scolastico ed extrascolastico.

Parole chiave: media education, social network, adolescenti, progetti educativi. 


\section{INTRODUZIONE}

Chi siamo nell'odierna società caratterizzata dalla presenza delle tecnologie? In particolare, chi sono gli adolescenti di oggi? Qual è la loro vera identità? Essa rimane invariata all'interno e all'esterno della realtà virtuale? Che ruolo hanno i social network nella vita delle giovani generazioni?

Attualmente, nel cercare di comprendere chi sono realmente gli adolescenti, non è possibile sottovalutare l'influenza esercitata dai social network nelle loro esperienze di vita quotidiana; questi ultimi, infatti, sono alcuni degli strumenti principali da loro utilizzati per comunicare e per interrogarsi circa la loro identità in divenire.

In merito all'utilizzo crescente di tali piattaforme virtuali, si è aperto un dibattito sulle modalità di definire le giovani generazioni; attribuire una singola definizione a tale categoria sociale risulterebbe limitato, se non addirittura errato. È per questo che, negli anni, si è assistito a una molteplicità di denominazioni che, a seconda delle caratteristiche che si intendono evidenziare nei giovani dell'ultimo millennio, vengono preferite le une alle altre. Semplicemente offrendo un elenco delle espressioni probabilmente più diffuse, ci si trova così a parlare, per esempio, di Nativi digitali (Prensky, 2001), Screen generation (Rivoltella, 2006), Net Generation (Tapscott, 2011), Adolescenti navigati (Lancini, 2015), Generazione hashtag (Manca, 2016).

La natura dei social network li rende strumenti ibridi, in grado di presentare rischi e opportunità per i relativi giovani fruitori e non solo; per quanto riguarda i primi, tra i più conosciuti, è possibile fare riferimento al cyberbullismo e al sexting (Shariff, 2017), alle social mode (Lancini, 2015), all'identità fluida e al narcisismo mediatico (Riva, 2016), alla violazione della privacy, alle fake news, all'analfabetismo emotivo (Goleman, 1995). Se non usati in maniera appropriata, infatti, i social network si possono rivelare una minaccia per il singolo, poiché questi risulta essere continuamente esposto a rischi che possono arrivare a intaccare il suo riconoscimento, la sua reputazione a livello sociale: in poche parole, la sua persona.

Oltre ai rischi però, appare adeguato non tralasciare anche le opportunità. A proposito di questo aspetto, infatti, tali piattaforme virtuali, se usate in maniera consapevole e responsabile, sono in grado di offrire agli adolescenti numerosi vantaggi, sia a livello relazionale e sociale, sia di crescita personale e culturale. Esse, infatti, si fanno principali canali di diffusione delle informazioni indispensabili per l'inserimento all'interno della società e consentono di mantenere contatti con il grup- po sociale di appartenenza, anche al di là della distanza fisica. È una realtà virtuale, parallela all'esistenza reale, che mette in continua connessione parti che formano un tutto, generazioni e saperi diversi (Codeluppi, 2018).

Per questi motivi, interventi di media education, finalizzati ad indirizzare i giovani utenti ad un uso consapevole e adeguato di tali strumenti mediali, appaiono di fondamentale importanza. Inoltre, tali percorsi educativi possono rivelarsi maggiormente efficaci se riescono a inserirsi all'interno della realtà globale degli adolescenti, ossia se sono in grado di favorire una connessione tra il loro contesto scolastico ed extrascolastico.

\section{I SOCIAL NETWORK COME PONTE TRA SCUOLA ED EXTRASCUOLA}

La comunicazione, con l'avvento e l'evoluzione della tecnologia, ha ampliato le proprie modalità. Alle prime forme verbali e visive si è aggiunta la forma scritta e, in un secondo momento, una molteplicità di linguaggi mediali derivata dall'utilizzo di media diversi. Tale pluralismo e intreccio di linguaggi ha messo in luce la necessità di un'educazione alla comunicazione, orientata a garantire il miglioramento della capacità di stare nel dialogo e di stare «in rete». La comunicazione, finalizzata allo sviluppo del pensiero e della riflessione critica da parte degli interlocutori, acquista così un valore formativo, caratterizzata da sempre da un orizzonte intenzionale complesso che, ora più che mai, va applicato, va tradotto in pratiche educative, scolastiche e massmediatiche. La scuola, basandosi su una progettualità educativa, si presenta l'agenzia formativa principale in grado di assicurare un iter formativo che tenga conto dell'età dei soggetti coinvolti e delle tipologie di linguaggio a cui si fa riferimento (Cambi, 2010).

Considerando, nel presente contributo, i social network come mezzo di comunicazione e gli adolescenti come destinatari principali, si sottolinea la necessità di garantire un'educazione ai media all'interno del contesto scolastico. In linea generale, si va a definire la media education come quel processo educativo che conduce all'acquisizione delle competenze e delle conoscenze digitali necessarie a utilizzare i media mediante un approccio critico (Buckingham, 2006).

Nello specifico, all'interno delle scuole, la media education rischia continuamente di essere considerata non come parte integrante del curriculum scolastico disciplinare, ma solamente come elemento trasversale. Delegando ogni docente all'utilizzo dei media nel corso delle proprie lezioni, infatti, essa finisce spesso per essere intesa come semplice utilizzo delle tecnologie per svol- 
gere le attività didattiche, andando così a perdere il suo vero significato e, di conseguenza, la sua valenza educativa (Grion \& Manca, 2016; Buckingham, 2020). Per conservare il suo scopo educativo, è opportuno concepirla come un continuo alternarsi tra l'approccio teorico e quello pratico, tra la cultura trasmessa e la creatività dei fruitori, tra l'analisi, la comprensione e la produzione dei mezzi e dei messaggi mediali (Ardizzone \& Rivoltella, 2008). Attraverso tale alternanza, essa viene intesa come elemento aggiuntivo all'estensione della trasmissione del sapere scolastico che, in questo caso, può essere garantito più facilmente anche al di fuori delle mura scolastiche; in tali circostanze, la media education si presenta come un punto di incontro tra il contesto formale della scuola e quello informale dell'extrascuola.

Estendere i confini della scuola alla realtà virtuale determina l'avvenire di una scuola digitale, la quale non deve essere intesa come scuola parallela, ma un contesto di apprendimento dato dalla fusione tra discipline scolastiche e interessi di vita reale maturati dagli studenti (Felini, 2008).

In Italia, la scuola digitale, come la si intende attualmente, è il risultato di un lungo percorso iniziato con l'introduzione dei media nella scuola agli inizi del Novecento: diapositive, filmine e poi, via via, la radio e la televisione (Felini, 2015; Galliani, 2002; Ranieri, 2011; Varisco \& Grion, 2000). La scuola digitale, quindi, è frutto di un processo che si è dibattuto tra diverse esigenze, quali il desiderio di rendere più rapido l'insegnamento, la necessità di rivolgersi a gruppi studenteschi sempre più ampi, il controllo politico sui contenuti da trasmettere, il voler far acquisire senso critico nei confronti dei linguaggi audiovisivi, fino alle prese di coscienza sui temi della "competenza digitale" (Calvani et al., 2009) e dell'Educazione Civica Digitale (MIUR, 2015; MIUR, 2020). Infatti, la scuola digitale promossa oggi si sviluppa da un'idea rinnovata di scuola, intesa non unicamente come luogo fisico limitato da confini tangibili, ma come spazio aperto per garantire anche lo sviluppo delle competenze utili per l'intera esperienza di vita. Secondo tale prospettiva, le tecnologie diventano ordinarie e abilitanti, al servizio dell'attività scolastica e di tutte le attività finalizzate alla formazione, ma anche estese a tutti gli spazi condivisi (classi, laboratori, ecc.) e informali della realtà territoriale (MIUR, 2015). Gli obiettivi del processo di insegnamento e apprendimento non mutano, ma vengono costantemente modificati per quanto riguarda le modalità e i contenuti trasmessi, per rispondere alle sfide evolutive, le quali richiedono competenze trasversali e un ruolo sociale attivo. Per raggiungere questo scopo, è necessario che il personale scolastico, gli studenti e l'organizzazione dei vari istituti di formazione siano continuamente disposti ad adattarsi alle sfide metodologico-didattiche provenienti dal contesto sociale, che vedono nell'innovazione la sua caratteristica principale.

L'uso dei social network, infatti, può garantire l'unione tra l'ambiente scolastico e la dimensione sociale e ludica della realtà informale. L'ibridazione che ne deriva risulta di fondamentale importanza e deve prevedere una certa tipologia di educazione all'interno della realtà virtuale, la quale, genericamente, viene definita "Netiquette», data dall'unione tra "network» (rete) ed «etiquette» (buona educazione). Essa sta ad indicare l'insieme di regole che cercano di disciplinare il comportamento di un utente nei confronti degli altri, all'interno del contesto digitale nel quale è inserito (Fogarolo, 2013, p. 103).

Scuole tradizionali che prevedono attività di educazione ai media, nello specifico attività di educazione ai social network all'interno del tradizionale curriculum scolastico, consentono di sperimentare quelle che possono essere definite «scuole social», ossia ambienti formativi ed educativi, in grado di adattarsi alle esigenze evolutive degli adolescenti, consentendo loro di accedere alle piattaforme online (social network, forum, blog, ecc.) che essi utilizzano quotidianamente, per svolgere anche attività didattiche e comunicative (Lancini, 2015, pp. 164-166).

Il PNSD, infatti, evidenzia come non sia più sufficiente parlare solamente di digitalizzazione all'interno del sistema scolastico perché, come sopraccitato, si rischierebbe di rimanere concentrati sulla dimensione tecnologica invece che su quella epistemologica e culturale; a fronte di questa consapevolezza, i social network vengono fatti rientrare all'interno di quell'educazione ai media che si intende garantire agli studenti, ponendosi come obiettivo la sfida dell'innovazione continua della scuola (MIUR, 2015).

Alcuni studi (Greenhow et al., 2014) evidenziano come i ragazzi, con particolare riguardo per gli adolescenti, siano sempre più desiderosi di sposare le pratiche partecipative dei social media e dei social network con quelle scolastiche o comunque legate alla scuola. Secondo tali contributi, si tratterebbe proprio di superare il confine tradizionale tra impegno sociale e civile e attività curriculari legate al ruolo scolastico. Ne consegue, dunque, il preciso compito degli insegnanti e della scuola di creare i contesti adeguati, invitando i giovani a rivestire il ruolo di co-autori e co-responsabili dei processi che si svolgono nelle comunità formative, luoghi che, per loro essenza, dovrebbero rappresentare la culla per lo sviluppo della democrazia (Grion \& Manca, 2015).

Una relazione educativa, appassionata e reciprocamente sostenuta, tra insegnanti e studenti, educatori e 
giovani, genitori e figli, può rivelarsi una risorsa e una modalità per riconoscere e valorizzare le molteplici capacità appartenenti alle due generazioni considerate: adolescenti e adulti.

\section{PROGETTI DI EDUCAZIONE AI SOCIAL NETWORK PER GLI ADOLESCENTI IN ITALIA: LA RICERCA ESPLORATIVA}

Al fine di esaltare l'importanza di interventi educativi finalizzati ad un'educazione ai social network, indirizzati agli adolescenti, è stata svolta una ricerca riguardo ai progetti che sono stati attuati e documentati tra il 2010 e il 2019 in diverse scuole secondarie e, alcuni di questi, estesi anche in contesti extrascolastici.

Di tale ricerca si esporrà la metodologia seguita, verranno descritti i progetti educativi reperiti e, infine, verrà proposto un confronto critico. Ovviamente, le conclusioni e le riflessioni maturate non riescono a rappresentare il fenomeno generale, ma possono ugualmente essere considerate utili al fine di mostrare differenti approcci seguiti e una certa evoluzione storica nel periodo di tempo considerato a riguardo delle metodologie adottate e della priorità attribuita alle tematiche affrontate nel corso degli anni.

\subsection{Finalità e metodologia della ricerca}

La ricerca dei progetti educativi è stata svolta adottando un approccio di tipo esplorativo (Corbetta, 2014): si è ritenuto opportuno ricercare dei progetti, realmente attuati, documentati e finalizzati all'educazione ai social network e destinati agli adolescenti.

I principali criteri di ricerca adottati per reperire i progetti sono stati:

- tipologia di progetti: educativi, escludendo progetti di ricerca.

- target: i destinatari dei progetti educativi sono gli adolescenti, nello specifico studenti dai 12 ai 18 anni;

- contesto: scuole secondarie;

- periodo temporale: 2010-2019;

- territorio di riferimento: Italia.

Decidendo, in un primo momento, di circoscrivere il campo di ricerca a livello nazionale, è stata riscontrata una certa difficoltà a reperire un numero consistente di progetti realizzati, in quanto la tematica considerata, appartenendo ad anni recenti, ha messo in luce una scarsa considerazione o documentazione dei percorsi educativi attuati da parte delle istituzioni formative ed educative nazionali. A questo punto, si è deciso di estendere la ricerca a livello internazionale, utilizzando la banca dati "Education Research Complete" di EBSCO, servendosi delle seguenti parole chiave in lingua inglese: adolescents, teenagers, social network, educational projects, media education, school.

I risultati emersi hanno messo in luce un numero ridotto di progetti educativi, ovvero otto, corrispondenti ai criteri di ricerca adottati. Invece, in numero maggiore sono risultati i progetti che ponevano i social network come argomento principale, ma presentavano scopi non educativi, ma di ricerca. Allo stesso tempo, molti progetti di educazione ai social network rilevati erano indirizzati ai bambini dai sei ai dieci anni e non agli adolescenti.

Tenuto conto della finalità della ricerca, dunque, si è deciso di restringere il campo, escludendo i percorsi educativi e di ricerca internazionali rilevati e di considerare solo i progetti realizzati a livello nazionale reperiti inizialmente, anche se in numero non elevato.

Per individuare i progetti nazionali, quindi, è stato utilizzato Google come motore di ricerca nel web e la banca dati EBSCO. Oltre alla ricerca libera, sono state consultate due riviste scientifiche online Form@re. Open Journal per la formazione in rete e Media Education. Studi, ricerche, buone pratiche», che, tra le altre disponibili, anche autorevoli, sono sembrate particolarmente attente ai temi dell'educazione ai media (Figura 1).

Il periodo dedicato alla ricerca si è sviluppato tra febbraio 2017 e novembre 2019. La ricerca è stata ripetuta più volte nell'arco del suddetto periodo di tempo.

Alla fase iniziale di ricerca è seguita quella di studio, stabilendo dei criteri di valutazione con lo scopo di poter classificare i progetti, andando ad analizzarli singolarmente in un primo momento, e, in un secondo momento, andando a fare un confronto tra questi.

Dunque, per ogni progetto, l'attenzione è stata rivolta principalmente a:

1. Fonte e anno di realizzazione;

2. Luogo di attuazione;

3. Ente responsabile della realizzazione;

4. Destinatari;

5. Finalità;

6. Obiettivi;

7. Strategie didattiche utilizzate;

8. Punti di forza;

9. Punti di criticità.

Facendo riferimento a tali criteri, i primi sono riconducibili a una descrizione delle caratteristiche proprie dei progetti, mentre gli ultimi due (8 e 9) sono frutto di una riflessione critica maturata a riguardo da parte di chi scrive e consistente nelle conclusioni tratte a seguito dell'analisi compiuta a confronto con la letteratura scientifica di riferimento. 


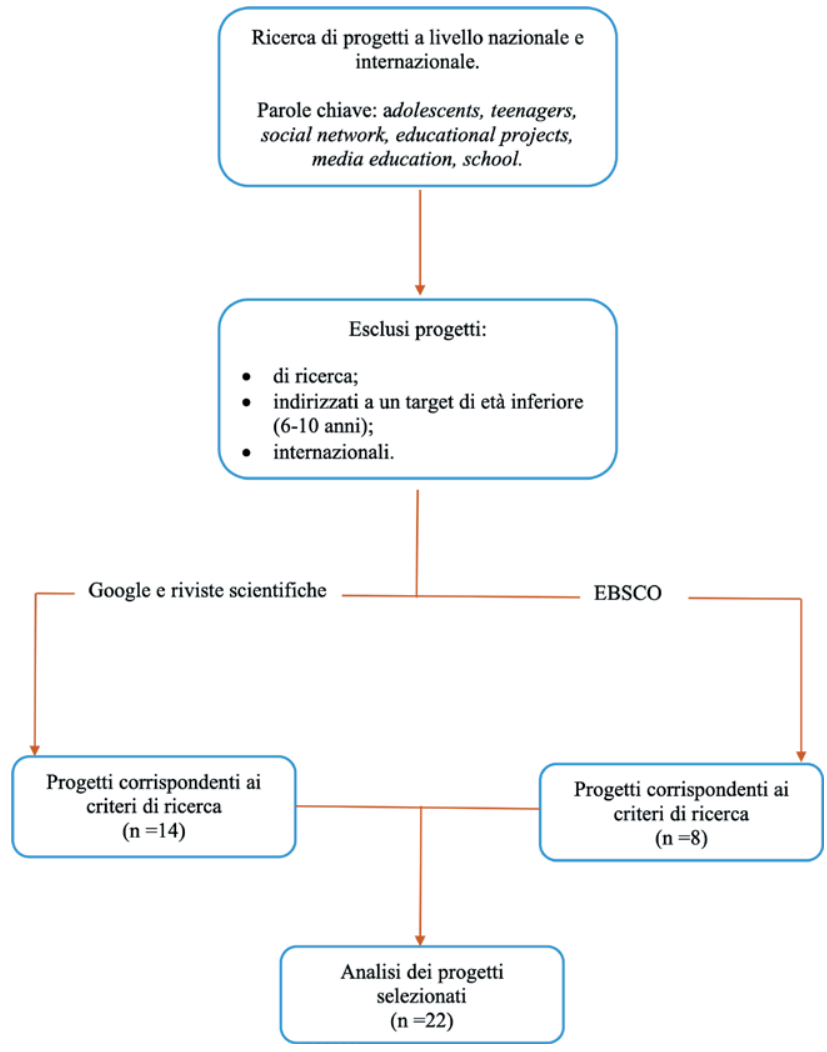

Figura 1. Diagramma di flusso del processo di ricerca.

\subsection{Presentazione dei progetti educativi selezionati}

I progetti educativi reperiti sono in totale $22 \mathrm{e}$, sulla base dell'ordine cronologico di pubblicazione, sono riportati nella Tabella 1.

In linea generale, $\mathrm{i}$ progetti considerati offrono alcuni esempi di come, attraverso attività e interventi di diverso tipo, agli adolescenti siano stati presentati sia i rischi che le opportunità che possono derivare dall'uso dei social network; un'attenzione particolare è stata rivolta al voler conoscere e comprendere le motivazioni principali che stanno alla base dell'utilizzo delle varie piattaforme da parte della tipologia di fruitori presa in considerazione (cfr. Grion \& Bianco, 2016; Manca, 2018). Nel complesso, i vari interventi educativi vanno ad affrontare alcuni temi emergenti rispetto all'uso dei social network, quali l'identità digitale, la sicurezza digitale, il modo di intendere le relazioni digitali e l'intenzione di far sviluppare le competenze mediali.

Facendo riferimento ai criteri di valutazione enunciati nel paragrafo precedente, nella Tabella 2, in allegato, si riportano singolarmente i 22 progetti sopraelencati, indicando le caratteristiche principali di ognuno di essi,
Tabella 1. Elenco dei progetti educativi reperiti e analizzati.

\begin{tabular}{|c|c|c|c|}
\hline & $\begin{array}{l}\text { Anno di } \\
\text { ubblicazione }\end{array}$ & Autori & Titolo \\
\hline 1 & 2010 & $\begin{array}{c}\text { Difensore civico \& } \\
\text { Corecom - ER }\end{array}$ & La rete siamo noi \\
\hline 2 & 2011 & Bruni \& Martini & Piagge mobili \\
\hline 3 & 2012 & Pedata \& Interlandi & Oltre lo schermo \\
\hline 4 & 2012 & Costa et al. & TWLetteratura \\
\hline 5 & 2012 & $\begin{array}{c}\text { Ufficio Garante } \\
\text { dell'Infanzia e } \\
\text { dell'Adolescenza FVG }\end{array}$ & Usale, non farti usare! \\
\hline 6 & 2013 & Sansone et al. & AAA Futuro Cercasi \\
\hline 7 & 2013 & Anonimo & Occhi in rete \\
\hline 8 & 2013 & Falconi \& Tirotta & Nord-Sud-Ovest-Web \\
\hline 9 & 2014 & Meneghetti & $\begin{array}{l}\text { Da laboratori con gli } \\
\text { studenti sui social } \\
\text { network ad una proposta } \\
\text { di loro uso didattico }\end{array}$ \\
\hline 10 & 2014 & Di Palo \& Papa & Play Tech \\
\hline 11 & 2014 & Anonimo & Una vita da social \\
\hline 12 & 2014 & Corecom-ER & Relazioni per crescere \\
\hline 13 & 2015 & Corecom-ER & A scuola con i media \\
\hline 14 & 2015 & Corecom-ER & Educare alla rete \\
\hline 15 & 2015 & Scuola di Robotica & Firewall \\
\hline 16 & 2015 & Corecom-ER & $\begin{array}{l}\text { La rete ti pesca. Usa la } \\
\text { testa }\end{array}$ \\
\hline 17 & 2015 & Anonimo & Smart Generation \\
\hline 18 & 2016 & Anonimo & $\begin{array}{c}\text { Come animare (e } \\
\text { innovare) la scuola...a } \\
\text { colpi di tweet! }\end{array}$ \\
\hline 19 & 2016 & Anonimo & E-likeschool \\
\hline 20 & 2016 & Sicurello & $\begin{array}{l}\text { Un percorso di media } \\
\text { education nella scuola } \\
\text { secondaria }\end{array}$ \\
\hline 21 & 2017 & Montanari & Sfida Social Zero \\
\hline 22 & 2019 & $\begin{array}{c}\text { Cooperativa Sociale } \\
\text { Tantintenti }\end{array}$ & Ops! \\
\hline
\end{tabular}

utili per rendere più comprensibile il confronto e la valutazione complessiva presentati nel paragrafo seguente.

\section{CHI? COME? PROGETTI DI MEDIA EDUCATION IN ITALIA}

In seguito all'analisi dei progetti educativi, la discussione dei risultati può essere avviata prendendo in considerazione i criteri di valutazione stilati nel momento iniziale della ricerca. In questo modo, è possibile mettere in evidenza, in maniera chiara e comprensibile, gli aspetti in comune e quelli che, invece, contraddistinguono alcuni progetti da altri. 
Le considerazioni emerse facendo riferimento ai singoli criteri utilizzati e il confronto finale tra i 22 progetti educativi consentono di cogliere soprattutto un'alternanza di metodologie adottate nel corso degli anni, la diversità nel trattare determinate tematiche e tralasciarne altre, il valore aggiunto o l'alta pericolosità che vengono attribuiti ai social network e la rilevanza affidata a un coinvolgimento attivo dei giovani.

\subsection{Distribuzione temporale e territoriale dei progetti}

A partire dal criterio di valutazione relativo all'anno, la ricerca è stata limitata volontariamente all'ultimo decennio. In alcuni casi la durata del progetto è stata prevista per un intero anno scolastico, mentre per altri si è limitata ad alcuni mesi, o addirittura, a singole giornate.

Se si considera il luogo, invece, i progetti realizzati risultano uniformemente diffusi a livello nazionale, con una prevalenza di Roma tra le città: sei progetti su 20. Volendo soffermarsi sulla distinzione tra Nord, Centro e Sud della penisola italiana, i percorsi educativi risultano essere stati attuati in numero simile nel Nord e nel Centro Italia: nove progetti su 20 nel primo caso e otto nel secondo. In particolare, tra le regioni del Nord, l'EmiliaRomagna è la regione predominante rispetto alle altre: quattro su nove progetti. Per quanto concerne il Centro Italia, è la Regione Lazio a prevalere, dal momento che Roma, come detto, è la città dove è stata realizzata la maggior parte dei progetti selezionati. Nel Sud Italia, invece, sono solamente due i progetti attuati tra quelli considerati: nello specifico, nelle città di Favara, Bari e Palermo. Tra i 22 progetti, due risultano essere stati realizzati in più regioni, quindi, non circoscritti al territorio di ideazione, ma diffusi in regioni del Nord, del Centro e del Sud Italia. Inoltre, il progetto "Smart Generation" risulta esteso a livello europeo.

\subsection{Enti promotori e destinatari dei progetti}

Prendendo in esame gli enti promotori dei vari progetti, tra i principali e maggiormente significativi, emergono: il MIUR, responsabile di tre progetti; Google; Telefono Azzurro; la Polizia Postale; Facebook; i vari Corecom regionali.

Gli enti elencati dimostrano l'importanza percepita da enti pubblici e privati del settore nell'implementare percorsi educativi con gli adolescenti, dal momento che i fenomeni di cyberbullismo, sexting, diffusione di fake news, furti di identità e altri appaiono come problematiche sociali in numero crescente.
Il fatto di non limitare gli interventi educativi all'interno di singoli istituti scolastici, ma di diffonderli in modo da creare una rete educativa in continua espansione, comporta il coinvolgimento dell'intera generazione adolescenziale. Inoltre, secondo una riflessione emersa in seguito all'analisi dei progetti selezionati, gli adolescenti, a loro volta, potrebbero fungere da modello per le generazioni successive, anche inconsapevolmente, per quanto riguarda i comportamenti da assumere e quelli da evitare in rete. Nella maggior parte dei progetti analizzati è stata praticata un'educazione di tipo intergenerazionale, in quanto, con l'obiettivo di ridurre il digital divide, sono state coinvolte le famiglie e, all'interno di esse, gli adolescenti sono stati considerati come «mediatori naturali» in termini di cultura digitale. Allo stesso modo, potrebbero essere attuati interventi formativi specifici basati sulla peer education, i quali potrebbero rivelarsi anche maggiormente efficaci rispetto a quelli tenuti da adulti formatori, sicuramente competenti, ma pur sempre adulti e spesso «temuti» dai giovani.

La collaborazione frequente con le diverse università italiane appare rilevante ai fini della progettazione di interventi di educazione ai social network, in quanto il coinvolgimento di docenti, appartenenti specialmente a corsi di laurea in Scienze della Formazione, Scienze dell'Educazione, Psicologia e Scienze della Comunicazione, permette di godere di un'adeguata supervisione scientifica.

Degna di nota appare la collaborazione con diversi paesi europei stabilita nel corso del progetto «Smart Generation», consentendo l'attuazione di quest'ultimo anche in Belgio, Spagna, Lettonia, Romania.

Per quanto concerne la tipologia dei destinatari ai quali sono stati indirizzati i progetti analizzati, il target ovviamente è lo stesso, poiché imposto dai criteri di ricerca volutamente stabiliti, ma rispetto alla categoria adolescenti, si è prevista una suddivisione: studenti delle scuole secondarie di $1^{\circ}$ e $2^{\circ}$ grado (dieci progetti); studenti delle scuole secondarie di $1^{\circ}$ grado (tre progetti); studenti delle scuole secondarie di $2^{\circ}$ grado (sette progetti) e studenti di scuole di ogni ordine e grado, comprese quindi le scuole primarie e dell'infanzia (due progetti).

La considerazione dei giovani a partire dall'età di 12 anni si rivela un'ottima strategia per l'efficacia dei progetti, dal momento che, nonostante l'età di iscrizione secondo il regolamento all'interno dei social network sia fissata a 14 anni, funge da strumento di prevenzione ai vari rischi e alla creazione di un'identità falsa.

Ai destinatari delle scuole primarie e dell'infanzia, invece, sono stati destinati progetti per iniziare ad approcciarsi al linguaggio e al mondo virtuale. 
Tra i destinatari, alcuni progetti prevedono anche i genitori (12 progetti) e gli insegnanti e/o educatori (sei progetti).

\subsection{Finalità, obiettivi e metodologie dei progetti}

Considerando la finalità, invece, emerge che la maggior parte dei progetti (12 progetti) persegue l'educazione a un uso appropriato dei social network o dei media in generale, dove per appropriato si intende un utilizzo corretto e responsabile di tali strumenti.

Interessante è anche la finalità del progetto educativo "AAA Futuro Cercasi", dal momento che esso considera i social network, in particolare Facebook e LinkedIn, come strumenti per promuovere l'orientamento universitario e lavorativo.

Se si analizza il progetto "Sfida Zero Social", invece, la finalità prevede di far cogliere agli adolescenti l'influenza che i media hanno nella loro vita quotidiana. Viene incentivato il digiuno digitale come sfida alla quale partecipano sia gli studenti che il docente promotore del progetto.

Gli obiettivi dei progetti, invece, sono molto eterogenei e molteplici. In ogni caso, è possibile individuarne alcuni comuni a più progetti, come:

1. accrescere la consapevolezza dei rischi online: quattro progetti;

2. comprendere e sviluppare il linguaggio mediale: cinque progetti;

3. cogliere le opportunità offerte dai social network: quattro progetti;

4. cogliere il valore e l'intensità delle relazioni virtuali: due progetti;

5. estendere i confini fisici della scuola, considerando la realtà virtuale come spazio dove poter svolgere delle attività didattico-educative: cinque progetti.

Sulla base degli obiettivi evidenziati, appare come l'attenzione sia stata riposta principalmente sull'indirizzare gli adolescenti a cogliere le opportunità che i social network sono in grado di offrire. Per quanto riguarda i rischi e i pericoli della rete, invece, la maggior parte degli interventi educativi è stata organizzata secondo un'ottica di informazione e prevenzione; solo in numero limitato, come mezzi di risoluzione di problematiche già verificatesi.

Per quanto concerne le metodologie adottate, come per i criteri di valutazione precedenti, è possibile individuarne alcune in comune:

1. discussione e riflessione condivisa: sette progetti.

In questi casi, oltre alle ore riservate alle tradizionali lezioni frontali in aula, finalizzate alla trasmissione delle conoscenze circa la tematica, si è cercato di favorire la partecipazione attiva dei destinatari, ponendo loro delle domande per conoscere la loro opinione in merito agli argomenti trattati. Sulla base delle risposte date, è stato promosso il confronto guidato in gruppo.

2. creazione di un profilo social: nove progetti.

Con il fine di promuovere l'analisi e la comprensione del linguaggio mediale e di far cogliere alcuni dei rischi e delle opportunità offerte dalle piattaforme virtuali, alcuni progetti hanno previsto la creazione e il conseguente utilizzo di una pagina o di un gruppo all'interno di un social network scelto a priori (principalmente Facebook o Twitter).

3. analisi di casi: due progetti.

Questi progetti, "A scuola coi media" e "Un percorso di media education nella scuola secondaria", hanno reso noti alcuni rischi che possono derivare dall'uso scorretto dei social network partendo dall'analisi di reali storie di vita di adolescenti.

4. realizzazione di foto e video: cinque progetti.

La produzione e la pubblicazione di contenuti mediali su profili o pagine social appositamente ideate, hanno concesso di educare i destinatari verso una duplice prospettiva: da una parte, infatti, essi sono stati indirizzati a creare alcune tipologie di prodotti mediali, quali foto e/o video, seguendo precise indicazioni, non dal punto di vista tecnico, ma riguardanti il messaggio da trasmettere; dall'altra, per poter individuare in rete i loro prodotti, i destinatari sono stati coinvolti attivamente nella creazione di una pagina sui social network, potendo così giungere a conoscenza del reale funzionamento e delle opportunità offerte da questi ultimi.

In alcuni progetti, come per esempio "La rete siamo noi", "Usale, non farti usare!" e "Occhi in rete", è stato ritenuto più opportuno tenere lezioni frontali in aula, garantendo ampio spazio ai formatori e meno ai destinatari, in quanto la trasmissione di informazioni risultava sufficiente in vista del raggiungimento degli obiettivi prefissati. La maggior parte dei progetti, invece, si è basata sullo svolgimento di lezioni interattive, nel corso delle quali la partecipazione attiva dei destinatari ha assunto un'importanza rilevante. Tale partecipazione non è stata limitata a semplici conversazioni tra formatori e uditori, ma ha concesso loro di intervenire per esprimere la propria opinione e produrre personalmente contenuti mediali. Ricorrendo a questa metodologia, è stato possibile attuare un intervento di educazione ai media, sviluppata nelle sue tre dimensioni: comprensione, fruizione e produzione (Felini, 2004).

La discussione e la riflessione condivisa hanno concesso ai destinatari di sviluppare soprattutto le prime 
due dimensioni di competenza, in quanto il confronto collettivo ha determinato l'unione di interpretazioni diverse dei media per poi cercare di comprenderle e valutarle mediante un approccio critico.

La partecipazione attiva ha anche concesso di mettere in pratica alcune specifiche strategie didattiche, quali il role-playing, particolarmente evidente nel progetto "Sfida Zero Social", in cui il gioco di ruolo è avvenuto nel momento in cui alcuni studenti insieme al loro docente partecipavano al digiuno digitale e un'altra classe, collaborando con i rispettivi insegnanti, rendeva note le dinamiche sulla pagina Facebook, fungendo da osservatori.

La collaborazione tra coetanei, o tra colleghi nel caso di formazione agli insegnanti, indispensabile per svolgere le attività laboratoriali di gruppo programmate in alcuni dei progetti analizzati, può essere intesa come momento di peer education.

\section{CONCLUSIONI: ANALISI CRITICA E PROSPETTIVE FUTURE}

Gli ultimi due criteri di valutazione, i punti di forza e di criticità, come già enunciato nel paragrafo 2.1., rispecchiano non la consapevolezza espressa dagli autori delle varie esperienze, ma la sensibilità di chi scrive, maturata nel corso dell'analisi dei progetti stessi e dal confronto con la letteratura scientifica inerente la tematica della media education (Buckingham, 2020; Cambi, 2010; Felini \& Trinchero, 2015; Galliani, 2002; Rivoltella, 2001). Tali aspetti, infatti, vengono trattati in riferimento ai progetti considerati, ma possono essere generalizzati e considerati come spunti di riflessione anche in vista di interventi educativi futuri.

Per quanto riguarda i punti di forza, quello principale, già messo in luce anche da ricerche precedenti (cfr. Grion \& Manca, 2016), è stato attribuito alla partecipazione attiva dei destinatari che accomuna ben 19 progetti su 22. I tre progetti che rimangono esclusi, nello specifico i progetti "La rete siamo noi", "Usale, non farti usare!" e "Occhi in rete", hanno previsto la distribuzione di materiale a titolo informativo, campagne di sensibilizzazione e incontri formativi frontali, destinati in particolare a insegnanti e genitori per metterli al corrente delle tematiche che riguardano principalmente gli adolescenti, verso i quali si dovrebbero attuare le diverse iniziative proposte.

Un secondo punto di forza è stato individuato nella collaborazione prevista tra il contesto scolastico e l'insieme di servizi extrascolastici che caratterizzano il vivere quotidiano degli adolescenti (Santerini, 1998). La mag- gior parte dei progetti ha cercato di estendere l'intervento educativo anche oltre le ore scolastiche, in modo da coinvolgere anche le famiglie o gli amici che non sono compagni di classe, ma con i quali si condividono i diversi interessi tipici dell'età adolescenziale. L'apertura verso la vita extrascolastica degli adolescenti consente di considerare i social network come strumenti che fungono da ponte tra il contesto formale della scuola, ritenuto spesso come ambiente morale, e il contesto informale dell'extrascuola, invece etichettato solitamente come immorale (cfr. Grion \& Manca, 2016; Manca, 2018). In questo modo, è possibile giungere alla conclusione che $\mathrm{i}$ suddetti contesti frequentati dagli adolescenti non possono essere etichettati secondo la prospettiva del giusto o sbagliato ma, al contrario, consentono di intendere i social network come strumenti di comunicazione, di accrescimento del sapere e di divertimento. In particolare, i progetti "Smart Generation" e "Una vita da social" hanno presentato direttamente tra gli obiettivi la volontà di favorire la cooperazione tra scuola ed extrascuola e, all'interno delle attività, moduli specifici e differenziati per genitori, insegnanti e adolescenti.

Proseguendo con la rilevazione dei punti di forza, i quattro progetti, "Relazioni per crescere", "Firewall", "Occhi in rete" e "Ops!", hanno realizzato gli incontri educativi attraverso un'équipe multidisciplinare. Il punto di forza evidenziato consiste nell'aver garantito, da parte degli ideatori, un contributo specificamente competente sulle tematiche trattate, coinvolgendo direttamente un esperto in materia, che ha potuto fungere da punto di riferimento per i destinatari dell'intervento.

Un quarto punto di forza è stato individuato nell'aver attuato un adattamento dei vari interventi sulla base dell'età dei destinatari (Felini \& Trinchero, 2015) e ciò, nello specifico, ha caratterizzato i progetti "A scuola coi media" e "Smart Generation". L'adattamento delle attività ai destinatari può essere riconosciuto come ulteriore punto di forza anche nel progetto «Una vita da social», dove le attività sono state distinte non considerando l'età, ma il ruolo dei destinatari (genitori, studenti o insegnanti).

Sicuramente ha costituito un vantaggio, il quinto, ai fini dell'efficacia dei progetti, l'aver anticipato la progettazione degli interventi con un'analisi dei bisogni e di lettura di dati di ricerca in merito al target e alle tematiche affrontate. I progetti che si sono serviti di tale metodologia di progettazione sono "La rete siamo noi" e "Occhi in rete".

Infine, alcuni enti promotori hanno costituito di per sé un punto di forza per i progetti stessi, dal momento che sono enti conosciuti a livello nazionale e, per questo motivo, possono garantire più facilmente la realizzazio- 
ne di interventi di vario tipo e, inoltre, possono godere di una maggiore affidabilità; tali enti, per esempio, sono Google, Telefono Azzurro, Facebook e il MIUR.

Per quanto concerne i punti di criticità, invece, non sono state riscontrate insufficienze pienamente penalizzanti i progetti considerati, ma solo limiti minori. Nello specifico, sono punti di criticità che accomunano alcuni interventi educativi e sono stati individuati, per esempio, nelle tempistiche, spesso insufficienti per intraprendere in maniera adeguata il percorso educativo progettato. Nel caso di alcuni progetti, infatti, la trattazione delle tematiche e lo svolgimento delle attività organizzate, sono state limitate ad un unico incontro. La continuità del percorso risulta spesso il mezzo migliore per ottenere dei risultati considerevoli. In particolar modo, tale discorso vale per gli adolescenti, i quali, affrontando un periodo evolutivo di grande e ininterrotto cambiamento, necessitano di essere affiancati regolarmente.

Altro limite riscontrato in alcuni progetti consiste nell'essere stati circoscritti a singoli contesti, realizzati da singoli educatori e insegnanti, con un numero ridotto di studenti coinvolti. Tale punto di criticità è emerso particolarmente nel corso di interventi attuati in via sperimentale, quindi rivolti solo a una o due classi, ma poi non più riprodotti, nemmeno in caso di successo. Una scarsa documentazione dei progetti, purtroppo, non consente di comprendere i motivi di tali scelte.

La carenza della documentazione fornita può rappresentare un ulteriore punto di criticità, in quanto, impedendo di rispondere ai criteri di analisi stabiliti, che stanno alla base di un progetto formativo, quali gli obiettivi, la metodologia, le fasi di lavoro e i tempi di realizzazione e la descrizione delle attività (Felini \& Trinchero, 2015), non consente di replicare il progetto formativo. Diversamente, sono da apprezzare i percorsi educativi che, a priori e in concomitanza con la realizzazione dell'intervento, riportano una descrizione accurata e le fasi di sviluppo, compresi i risultati ottenuti, anche attraverso una pagina di un social network, creata appositamente e prevista come attività, o una piattaforma online. Un esempio considerevole di progetto altamente descritto e continuamente aggiornato è "Smart Generation".

Infine, un limite che, invece, non dovrebbe presentarsi, poiché si prendono in considerazione i social network che caratterizzano la quotidianità informale degli adolescenti, è il mancato coinvolgimento del contesto e dei servizi extrascolastici. Viceversa, un progetto limitatamente attuato solo nei contesti extrascolastici, senza collaborare con le scuole, rischia di far credere alle giovani generazioni che esista una sorta di separazione tra le due realtà, scolastica ed extrascolastica; maggiormente interessata al rendimento e al comportamento scolastico la prima e, invece, alla vita quotidiana la seconda. Trasmettere un'idea di continuità e di connessione tra i due ambiti potrebbe rivelarsi proficuo nel percorso di crescita dei giovani che, in questo modo, anche quando non lo richiedono, potrebbero sentirsi continuamente supportati.

In conclusione, ritengo che la ricerca, l'analisi e le considerazioni comparative e valutative qui esposte sui progetti considerati abbiano offerto una panoramica generale, seppur circoscritta a spazi e tempi precisi, circa le modalità di pensare e ideare interventi educativi sulla tematica affrontata. In particolar modo, le riflessioni conclusive, emerse in riferimento a quelli che sono stati delineati come punti di forza e di criticità, potrebbero rivelarsi utili per auspicabili progetti futuri, che tengano conto dell'identità dinamica, in continuo divenire, degli adolescenti e, parallelamente, dell'evolversi incessante delle piattaforme virtuali da questi utilizzate. Sulla base di ciò, per garantirne una maggior efficacia, interventi futuri di educazione sull'uso consapevole e corretto dei social network dovranno essere adeguatamente adattati dal punto di vista teleologico e metodologico, in base ai bisogni educativi presentati al momento da parte degli adolescenti. Una certa flessibilità potrà garantire un continuo adattamento del progetto ideato alle modalità di reazione e di coinvolgimento dei destinatari e delle condizioni del contesto in cui ci si troverà a intervenire.

Partire dagli interessi principali degli adolescenti, dal dare loro la possibilità di verbalizzare ciò che pensano e le sensazioni che sentono per capirli, comprenderli, e arrivare così a indirizzarli verso le opportunità e, di conseguenza, ad allontanarli dai rischi nei quali potrebbero imbattersi, dovrebbe essere l'obiettivo principale a cui aspirare quando si parla di progetti educativi di tale natura.

\section{RINGRAZIAMENTI}

Lautrice ringrazia, sentitamente, il professor Damiano Felini, docente di Pedagogia generale e sociale e Pedagogia dei media presso l'Università di Parma, in quanto prezioso collaboratore alla ricerca.

\section{RIFERIMENTI BIBLIOGRAFICI}

Ardizzone, P., \& Rivoltella, P.C. (2008). Media e tecnologie per la didattica. Vita e Pensiero.

Bruni, I., \& Martini, F. (2011). Piagge mobili. Mobile storytelling alla periferia di Firenze, Media Education. Studi, ricerche, buone pratiche, 2(2), 211-225. 
Buckingham, D. (2006). Media education. Alfabetizzazione, apprendimento e cultura contemporanea. Erickson.

Buckingham, D. (2020). Un manifesto per la media education. Mondadori Università.

Calvani A., Fini A., Ranieri M. (2009). Valutare la competenza digitale. Modelli teorici e strumenti applicativi. TD - Tecnologie Didattiche, 48, 39-46.

Calvani, A., Fini, A., Ranieri, M., \& Picci, P. (2012). Are young generations in secondary school digitally competent? A study on Italian teenagers. Computers \& Education, 58(2), 797-807. https://doi.org/10.1016/j. compedu.2011.10.004

Cambi, F. (a cura di) (2010). Media Education tra formazione e scuola. Principi, modelli, esperienze. Edizioni ETS.

Codeluppi, V. (2018). Il tramonto della realtà. Carocci.

Cook, J., \& Pachler, N. (2012). Online people tagging: Social (mobile) network(ing) services and workbased learning. British Journal of Educational Techno$\log y, 43(5), 711-725$. https://doi.org/10.1111/j.14678535.2012.01346.x

Cooperativa Sociale Tantintenti (2019). Progetto OPS! Per un uso consapevole dei social e delle reti web. https://percorsiconibambini.it/communityschool/2019/03/12/progetto-ops-per-un-uso-consapevoledei-social-e-delle-reti-web/ (ver. 01.04.2021).

Corbetta, P. (2014). Metodologie e tecniche della ricerca sociale. Il Mulino.

Corecom-ER (2015). A scuola coi media. https://www. assemblea.emr.it/corecom/le-attivita/vigilanza-e-controllo/educazione-ai-media-1/copy_of_educazioneai-media/iniziative-2015/a-scuola-coi-media_2013-1 (ver. 01.04.2021).

Corecom-ER, \& Difensore civico (2010). La rete siamo noi. Iniziative per un uso sicuro della rete Internet $e$ del cellulare da parte dei minori. http://www.assemblea.emr.it/corecom/attivita/educazione-ai-media/iniziative-2010/la-rete-siamo-noi (ver. 01.04.2021).

Corecom-ER (2014). Relazioni per crescere. Percorsi per l'uso consapevole dei media e la prevenzione del cyberbullismo.

http://www.assemblea.emr.it/corecom/attivita/educazione-ai-media/iniziative-2015/relazioni-per-crescere (ver. 01.04.2021).

Corecom-Umbria (2015). Educare alla rete.

http://www.consiglio.regione.umbria.it/educare-alla-rete (ver. 01.04.2021).

Corecom-Umbria (2015). La rete ti pesca. Usa la testa.

http://www.corecom.umbria.it/la-rete-ti-pesca-usa-latesta (ver. 01.04.2021).

Costa, P., Vaccaneo, P., Montenegro, E., \& Moscardi, I. (2012). TwLetteratura. https://www.twletteratura. org/2016/10/scuola/ (ver. 01.04.2021).
Di Palo, B., \& Papa, R. A. (2014). Play Tech: riattivare la comunicazione fra minori e genitori attraverso internet. Media Education. Studi, ricerche, buone pratiche, 5(2), 208-219.

Imprese di Talento (2015, Marzo 1). Educare e in-formazione all'uso del web e social media nelle scuole. Nasce e-likeschool (2016). www.impreseditalento. com/.../Progetto-Educazione-alluso-dei-SocialMedia-nelle-scuole/ (ver. 01.04.2021).

Falconi, A., \& Tirotta, R. (a cura di) (2013). Quaderno di educazione ai media. L'esperienza del Corecom EmiliaRomagna. https://www.centroalbertomanzi.it/quaderno-di-educazione-ai-media/ (ver. 01.04.2021).

Felini, D. (2004). Pedagogia dei media. Questioni, percorsi e sviluppi. Editrice La Scuola.

Felini, D. (2008). Crossing the Bridge: Literacy between School Education and Contemporary Cultures. In Flood, J., Brice Heath, S., \& Lapp, L. (a cura di), Handbook of Research on Teaching Literacy Through the Communicative and Visual Arts (pp. 19-26). New York-London. Erlbaum. https://doi. org/10.4324/9781315759616

Felini, D. (a cura di) (2015). Educare al cinema: le origini. Riflessioni ed esperienze di pedagogia dei media fino agli anni della contestazione. Guerini Scientifica.

Felini, D., \& Trinchero, R. (a cura di) (2015). Progettare la media education. Dall'idea all'azione nella scuola e nei servizi educativi. Franco Angeli.

Fogarolo, A. (2013). Do you speak Facebook? Guida per genitori e insegnanti al linguaggio del social network. Erickson.

Fondazione Roma Solidale (2013). Occhi in Rete. www. fondazioneromasolidale.it

Galliani, L. (2002). Appunti per una vera storia dell'educazione ai media, con i media, attraverso i media. Studium Educationis, 563-576.

Goleman, D. (1995). Intelligenza emotiva. Rizzoli.

Greenhow, C., Gleason, B., \& Li, J. (2014). Psychological, social, and educational dynamics of adolescents' online social networking. Media Education: Studi, Ricerche, Buone Pratiche, 5(2), 115-130.

Grion, V., \& Bianco, S., (2016). Social network come strumenti didattici: percezioni e atteggiamenti di insegnanti e studenti. TD Tecnologie Didattiche, 24(3), 136-146.

Grion, V., \& Manca, S., (2015). Voci e silenzi in un'esperienza di Student Voice mediata dai social network. TD Tecnologie Didattiche, 23(2),70-80.

Grion, V., \& Manca, S., (2016). Social network sites in secondary school: when students and teachers express their viewpoints. ICERI 2016 Proceedings, 5992-6002. https://doi: 10.21125/iceri.2016.0357 
Lancini, M. (2015). Adolescenti navigati. Come sostenere la crescita dei nativi digitali. Erickson.

Livingstone, S., Haddon, L., Görzig, A., \& Ólafsson, K. (2011). Risks and safety on the internet: the perspective of European children. Full Findings. LSE. EU Kids Online. 3-60.

Manca, M. (2016). Generazione hashtag. Gli adolescenti dis-connessi. Alpes Italia.

Manca, S. (2018). Social network sites in formal and informal learning: potentials and challenges for participatory culture. RiMe, 2, 77-88. https://doi. org/10.7410/1355

Meneghetti, C. (2014). Da laboratori con gli studenti sui social network ad una proposta di loro uso didattico. Bricks, 4(4), 100-113.

MIUR (2015). Piano Nazionale Scuola Digitale. https:// www.miur.gov.it/web/guest/scuola-digitale (ver. 01.04.2021).

MIUR (2016). Come animare (e innovare) la scuola...a colpi di tweet!

MIUR (2020). Linee guida per l'insegnamento dell'educazione civica. https://www.miur.gov.it/web/guest/-/ decreto-ministeriale-n-35-del-22-giugno-2020 (ver. 01.04.2021).

Montanari, L. (2017). Prato, una settimana di "digiuno digitale" per $i$ ragazzi di tre classi. La Repubblica. http://firenze.repubblica.it/cronaca/2017/05/14/news/ prato_una_settimana_di_digiuno_digitale_per_i_ ragazzi_di_tre_classi-165447316/(ver. 01.04.2021).

Pedata, L. T., \& Interlandi, M. (2012). Adolescenti e tecnologie ai "tempi della crisi": interventi educativi. Rivista Scuola IaD, Ricerca e tecnologia, 5.

Prensky, M. (2001). Digital Natives, Digital Immigrants Part 1. On the Horizon, 9(5), 1-6. https://doi. org/10.1108/10748120110424816

Ranieri, M. (2011). Le insidie dell'ovvio. Tecnologie educative e critica della retorica tecnocentrica. ETS.

Repubblica italiana (2015). Legge 13 luglio 2015, n. 107 in materia di «Riforma del sistema nazionale di istruzione e formazione e delega per il riordino delle disposizioni legislative vigenti».

Riva, G. (2010). I social network. Il Mulino.

Riva, G. (2014). Nativi digitali. Crescere e apprendere nel mondo dei nuovi media. Il Mulino.

Riva, G. (2016). Selfie. Narcisismo e identità. Il Mulino.

Rivoltella, P.C. (2001). Media education. Modelli, esperienze, profilo disciplinare. Carocci.

Rivoltella, P.C. (2006). Screen Generation. Gli adolescenti e le prospettive dell'educazione nell'età dei media digitali. Vita e Pensiero.

Sansone, N., Cucchiara, S., \& Ligorio, M. B. (2013). Lorientamento attraverso i social network: il proget- to “AAA Futuro Cercasi”. Form@re, 13(1), 81-90. https://doi.org/10.13128/formare-12617

Santerini, M. (1998). L'educatore tra professionalità pedagogica e responsabilità sociale. La Scuola.

Scuola di Robotica (2015). Firewall. Giovani pronti a navigare! https://firewall.scuoladirobotica.it/it/ homefw.html (ver. 01.04.2021).

Shariff, S. (2017). Sexting e cyberbullismo. Quali limiti per i ragazzi sempre connessi?. Edra.

Sicurello, R. (2016). Un percorso di media education nella scuola secondaria. Media Education. Studi, ricerche, buone pratiche, 7(1), 94-115. https://doi: 10.14605/MED711608

Smart Generation (2015). http://bit.ly/smartgeneration (ver. 01.04.2021).

Tapscott, D. (2011). Net generation. Come la generazione digitale sta cambiando il mondo. Franco Angeli.

Ufficio Garante dell'Infanzia e dell'Adolescenza Friuli Venezia Giulia (2017). Usale, non farti usare!.

https://ic-ronco.edu.it/sito-download-file/982/all (ver. 01.04.2021).

Una vita da social (2014). http://www.poliziadistato.it/ articolo/31696 (ver. 01.04.2021).

Varisco, B.M., \& Grion, V. (2000). Apprendimento e tecnologie nella scuola di base. UTET. 


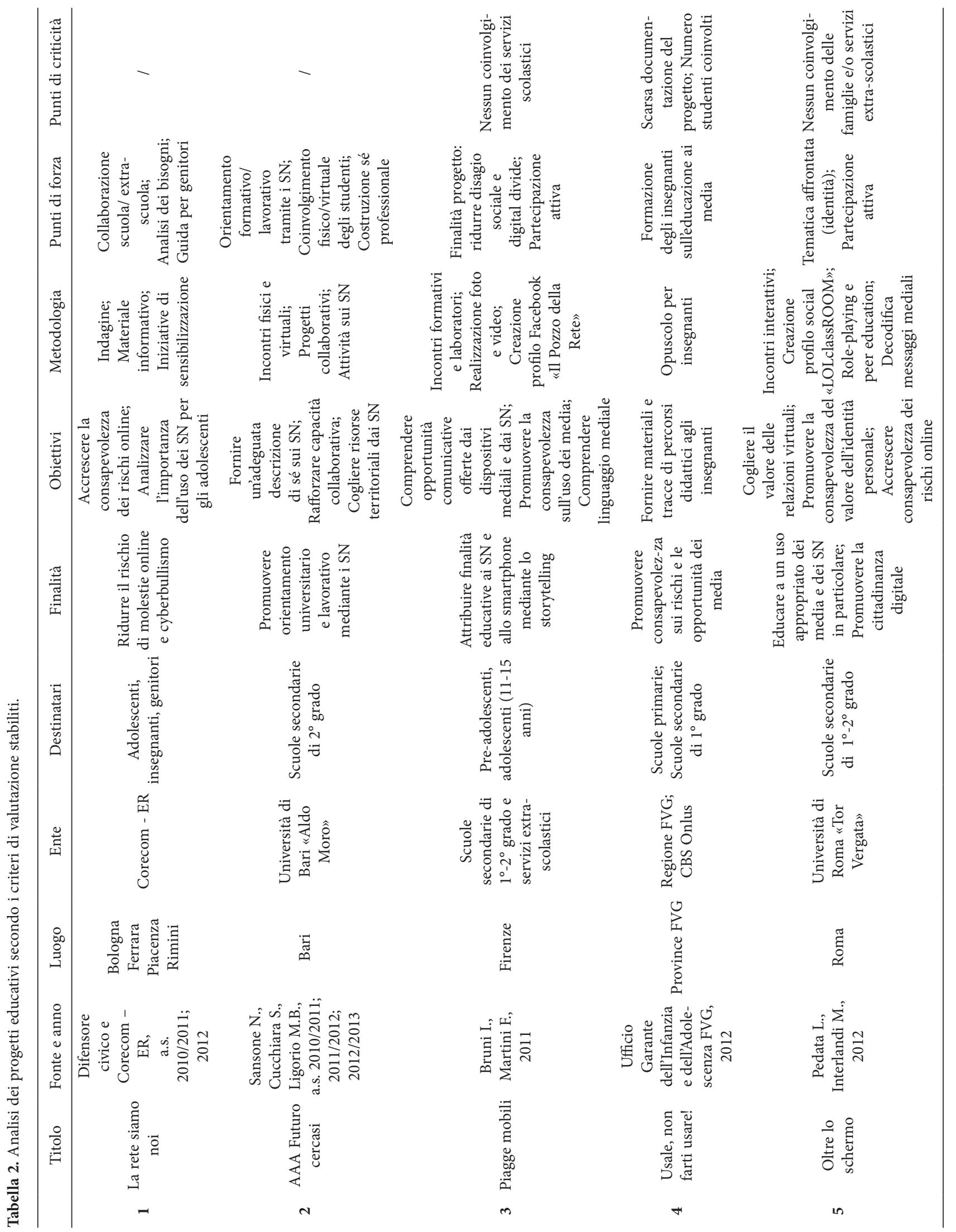




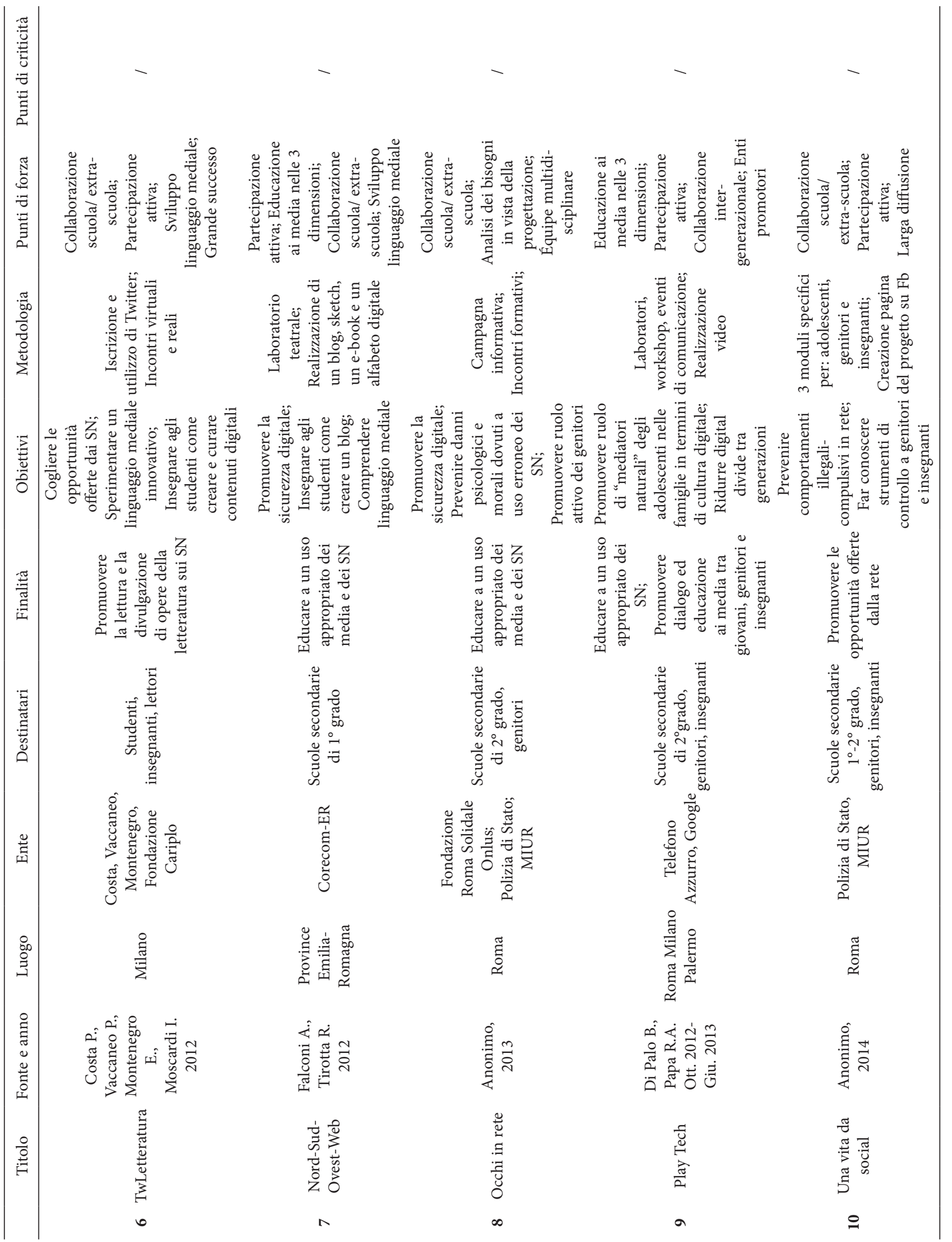




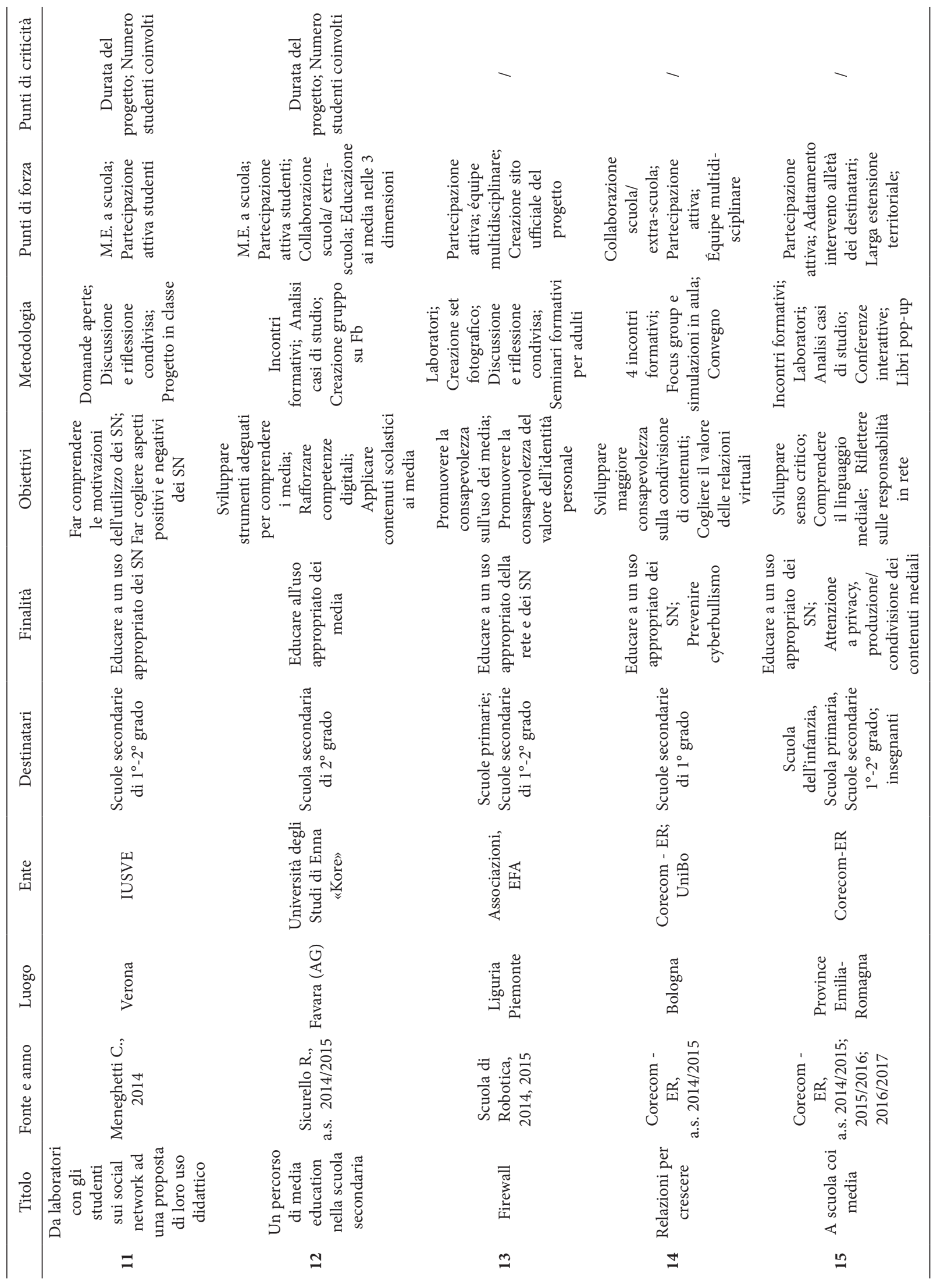




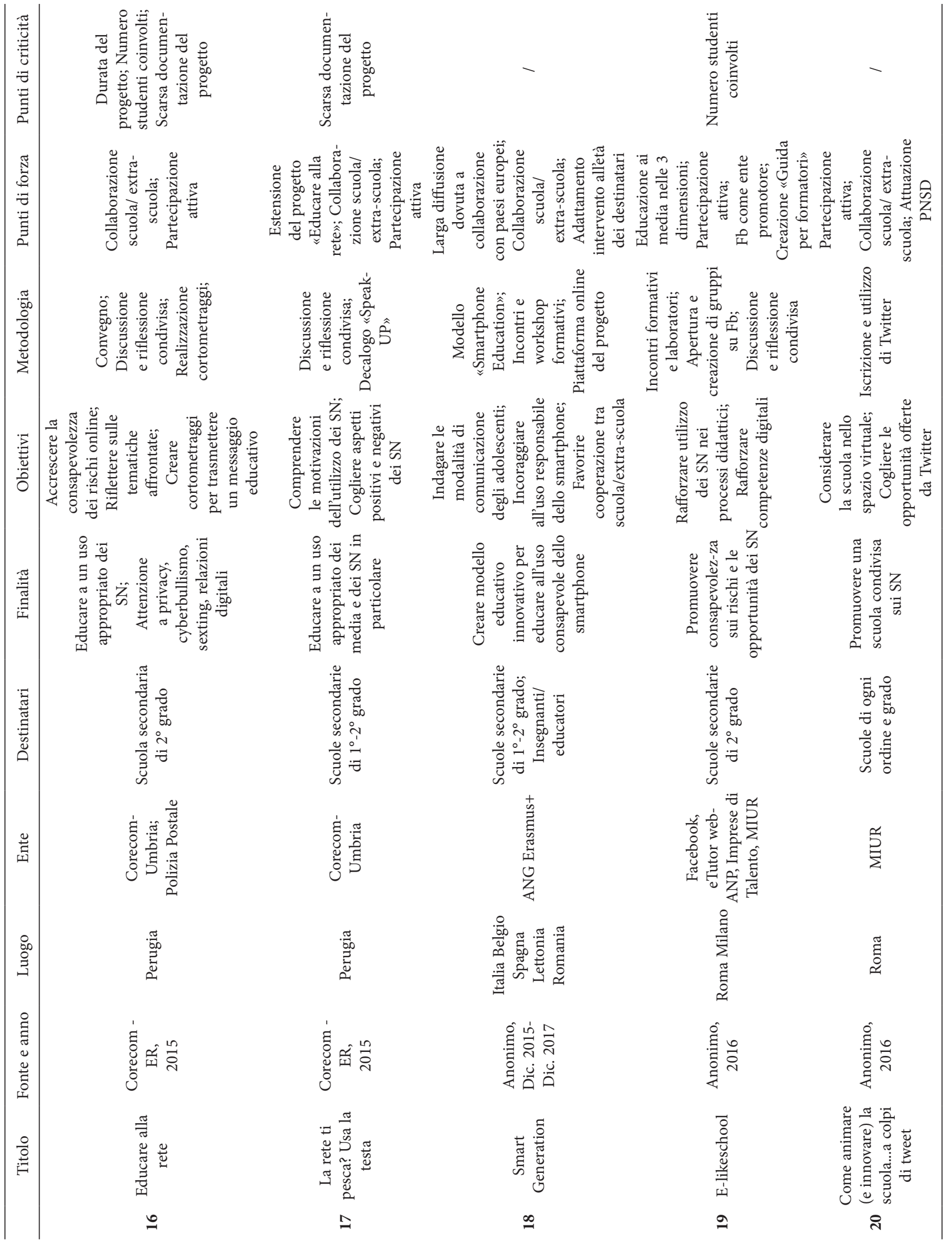




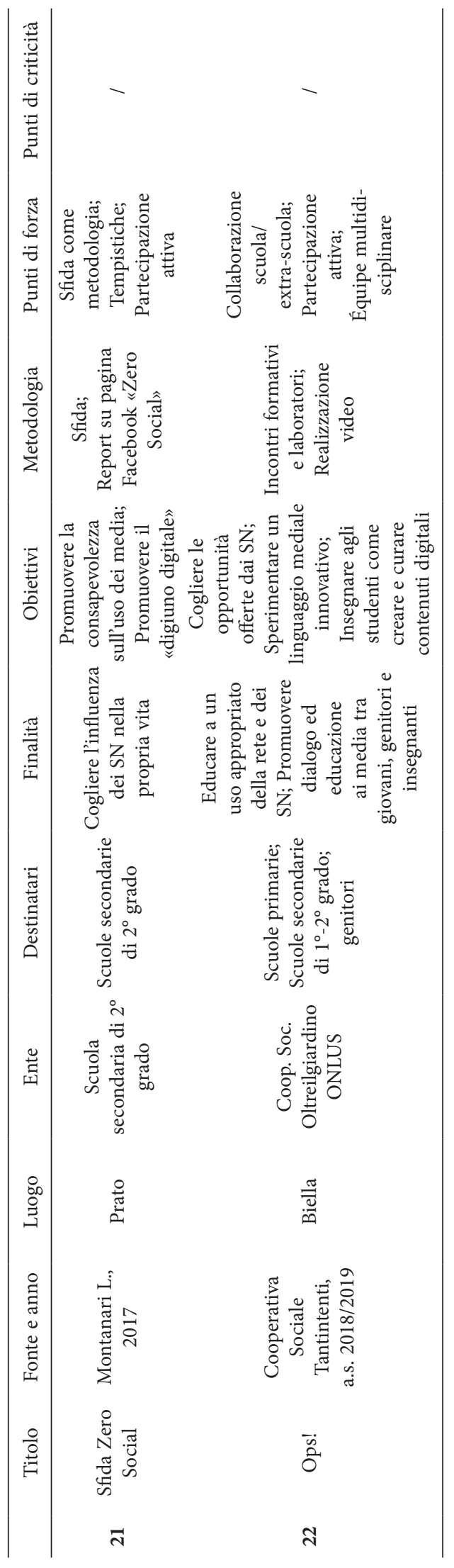

